\title{
Metallic Ternary Telluride with Sphalerite Superstructure
}

Amit Adhikary, ${ }^{\mathrm{a}}$ Sudip Mohapatra, ${ }^{\mathrm{a}}$ Seng Huat Lee, ${ }^{\mathrm{b}}$ Yew San Hor, ${ }^{\mathrm{b}}$ Puja Adhikari, ${ }^{\mathrm{c}}$ WaiYim Ching, ${ }^{\mathrm{c}}$ Amitava Choudhury, ${ }^{\mathrm{a}, *}$

${ }^{\mathrm{a}}$ Department of Chemistry, Missouri University of Science and Technology, Rolla, MO 65409, USA

${ }^{\mathrm{b}}$ Department of Physics, Missouri University of Science and Technology, Rolla, MO 654090010, USA

${ }^{c}$ Department of Physics and Astronomy, University of Missouri-Kansas City, Kansas City, MO 64110, USA 


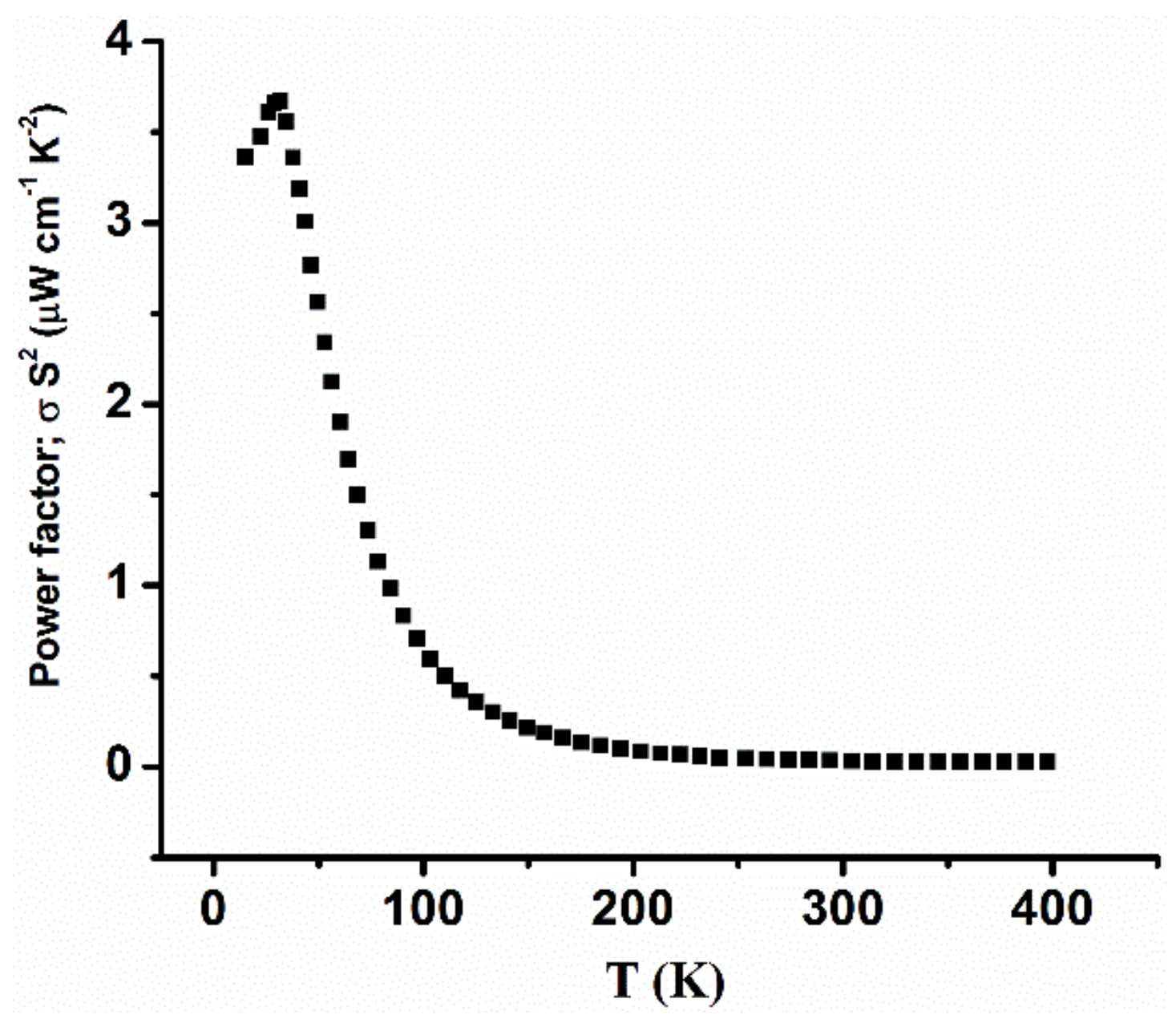

Figure S1. Temperature dependence of power factor of $\mathrm{Cu}_{5} \mathrm{Sn}_{2} \mathrm{Te}_{7}$. 


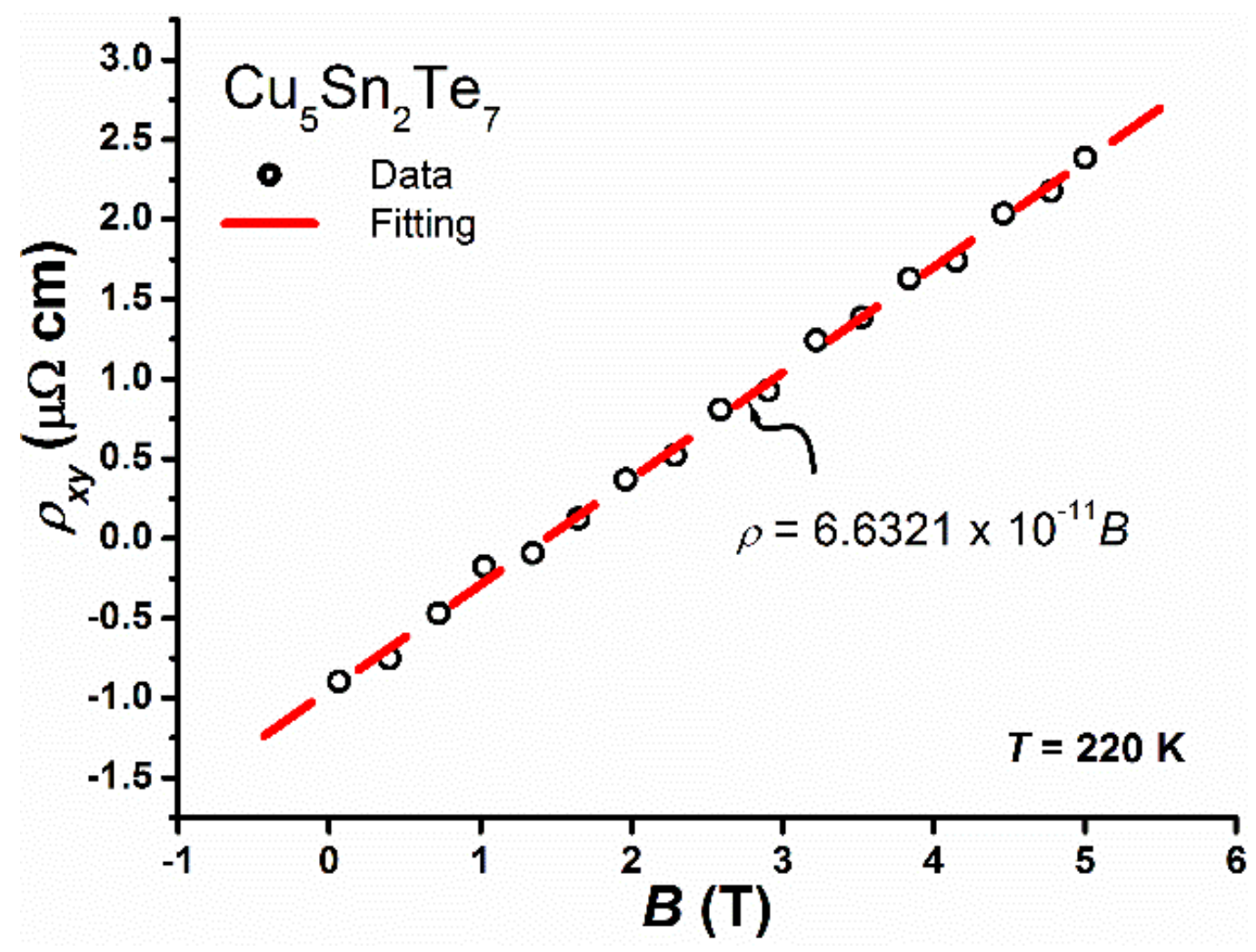

Figure S2. Hall resistivity $\rho_{\mathrm{H}}$ versus the magnetic field B at the temperature of $220 \mathrm{~K}$ for a sample of $\mathrm{Cu}_{5} \mathrm{Sn}_{2} \mathrm{Te}_{7}$ exhibits a linear relation with the magnetic field. 

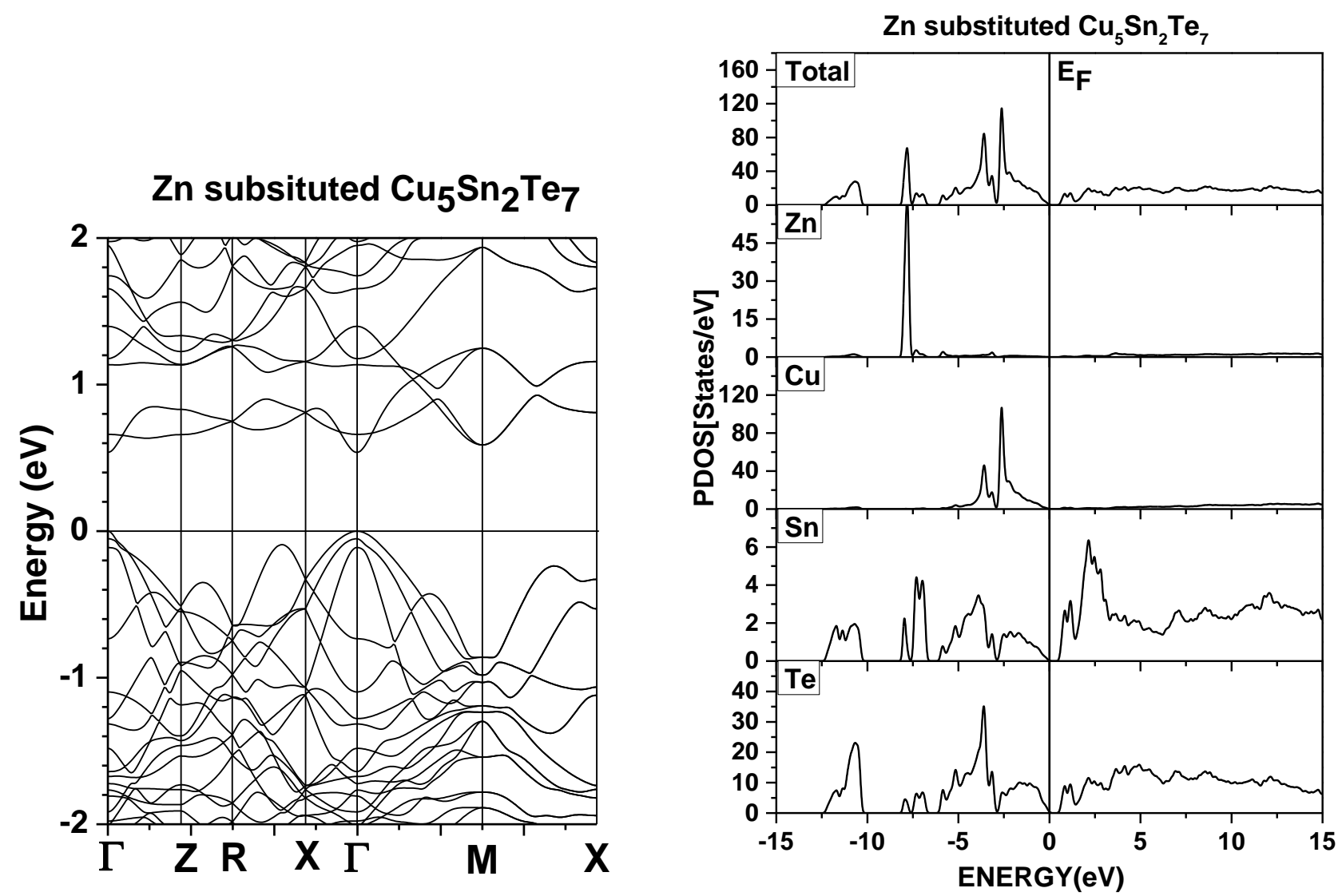

Figure S3. (a) Band structure of $\mathrm{Cu}_{4} \mathrm{ZnSn}_{2} \mathrm{Te}_{7}$. (b) The DOS and PDOS plots for $\mathrm{Cu}_{4} \mathrm{ZnSn}_{2} \mathrm{Te}_{7}$. 